\title{
Prevalence of Dry Eye Disease in Connective Tissue Disorders Among the Patients Attending a Tertiary Care Hospital in South Kerala - A Cross Sectional Study
}

\author{
Parvathy R. ${ }^{1}$, Kiran Balagopal ${ }^{2}$ \\ 1,2 Department of Ophthalmology, Travancore Medical College, Kollam, Kerala, India.
}

\section{ABSTRACT}

\section{BACKGROUND}

Dry eye diseases (DED) tend to be more seen in patients with auto immune disease. This study was an attempt to assess the prevalence of DED among the patients who were diagnosed and confirmed cases of Rheumatoid arthritis (RA), SLE and Primary Sjogren's syndrome (Primary SS). Objective of the study was to assess the prevalence of Dry eye disease among the study participants and determine its severity in connective tissue disorders.

\section{METHODS}

This was a cross sectional study conducted at a tertiary care hospital in the South Kerala. The study period was six months. 30 study participants were enrolled for the study who fulfilled the selection criteria. The study participants were evaluated for DED by Schirmer's test, Tear break up time and ocular surface staining. Data collected was entered in Microsoft Excel and descriptive data was expressed as frequencies and percentage.

\section{RESULTS}

The overall prevalence of the Dry eye disease was found to be $54 \%$. The prevalence was significantly higher in patients with Primary SS which accounted for $70 \%$ followed by patients with RA; which accounted for 52. Dry eye disease was seen only in $40 \%$ of the study participants diagnosed with SLE. Similarly, the severity of Dry eye disease was found higher in patients with Primary SS; where about $35 \%$ had severe to very severe disease. $22 \%$ of the study participants with RA had severe to very severe dry eye disease. There was no severe or very severe dry eye disease in the study participants who had SLE.

\section{CONCLUSIONS}

The overall prevalence of Dry eye disease in patients with autoimmune disease was found to be significantly higher especially in patients who were diagnosed to have Primary SS and RA. There is a need for early diagnosis of dry eye disease in such patients which can improve the cure rate, reduce the complications of the disease and reduce the visual morbidity.

\section{KEY WORDS}

Dry Eye Disease, Primary sjogrens Syndrome, Rheumatoid Arthritis, SLE
Corresponding Author:

Dr. Kiran Balagopal,

Travancore Medical College,

Kollam, Kerala, India.

E-mail:drkb2001@gmail.com

DOI: $10.14260 /$ jemds $/ 2022 / 51$

How to Cite This Article:

Parvathy R, Balagopal K. Prevalence of dry eye disease in connective tissue disorders among the patients attending a tertiary care hospital in south Kerala - a cross sectional study. J Evolution Med Dent Sci 2022;11(01):272-276, DOI: $10.14260 / \mathrm{jemds} / 2022 / 51$

Submission 04-12-2021,

Peer Review 12-12-2021,

Acceptance 29-01-2022,

Published 31-01-2022.

Copyright (C) 2022 Parvathy R. et al. This is an open access article distributed under Creative Commons Attribution License [Attribution 4.0 International (CC BY 4.0)] 


\section{BACKGROUND}

Dry eye is a disease of multiple etiologies of the tears and ocular surface which causes symptoms of visual disturbances, discomfort and instability of the tear film with potential damage to the ocular surface. ${ }^{1}$ It is a common yet less recognized condition which varies in the severity, duration and etiology. ${ }^{2}$ In most cases, the condition is not threatening and is characterized by mild irritation and blurring of the vision. Certain medications and environmental conditions trigger the disease. Elimination of such factors is of preventive as well as of curative value

Dry eye disease is often under diagnosed in patients with connective tissue disorders. There is a high prevalence of Dry eye disease in auto immune disorders such as Rheumatoid arthritis (RA), Sjogren's syndrome (SS) and Systemic lupus erythematosus (SLE). ${ }^{3}$ Patients presenting with systemic disease often ignore the symptoms associated with dry eye due to other worsened complications associated it, which lead to irreversible damage of the ocular surface and occurrence of severe dry eye manifestations in the latter stage of the disease.

Dry eye disease is categorized into two: Aqueous tear deficient dry eye (ADDE) and Evaporative dry dye. Aqueous tear deficient dry eye (Lacrimal tear deficiency) implies that the dry eye is due to the lacrimal tear secretion failure ${ }^{4}$. It is further divided into two: Sjogrens Syndrome dry eye and Non Sjogren syndrome dry eye.

Sjogren syndrome dry eye (SSDE) is an autoimmune condition in which the lacrimal and salivary glands are targeted, other organs also are affected. The salivary as well as lacrimal glands are infiltrated by the activated $\mathrm{T}$ cells which cause acinar and ductular cell death which in turn results in the decreased secretion of tears and saliva. Activation of the inflammatory agents within the glands results in the expression of auto antigens at the epithelial cell surface 4 and causes the retention of tissue-specific CD4 and CD8 T-cells. ${ }^{5}$ Secondary Sjogrens syndrome includes the features of primary SS along with the features of an autoimmune connective disease. ${ }^{6}$

Non-Sjogren syndrome dry eye is a type of ADDE caused due to lacrimal dysfunction, where the autoimmune characteristics of SSDE have been excluded. The commonest form is age-related dry eye, to which the term Kerato conjunctivitis (KCS) has sometimes been applied in the past.7

Evaporative dry eye is divided into intrinsic and extrinsic; in which intrinsic causes are mainly due to meibomian oil deficiency, lid aperture disorders, decreased blink rate and due to action of certain drugs such as accutane, whereas extrinsic causes of the evaporative dry eye is mainly caused due to Vitamin A deficiency. It is seen commonly in contact lens wearers and it is also associated with allergy. ${ }^{8}$

The treatment for dry eye aims in reducing the signs and associated symptoms such as ocular irritation, mucous discharge and redness. Early diagnosis and treatment aids in provision of improved visual function and in prevention of ocular surface damage.

Recent advances in the etiology of the dry eye disease pave the way for more opportunities in the early diagnosis as well as disease management. Early diagnosis and treatment of the underlying systemic immune condition significantly reduce the visual morbidity and even may be lifesaving for many patients. ${ }^{9}$

Early detection of dry eye disease can markedly improve the patients comfort and can also aid in minimizing the further ocular surface structural damage. ${ }^{10}$ This study aimed to assess the prevalence of dry eye disease among the patients presenting with connective tissue disorders such as Rheumatoid arthritis, Primary Sjogrens, SLE and to determine the severity of dry eye disease among the same connective tissue disorders.

\section{METHODS}

Institutional Ethics Committee approval and permission from the institutional head was obtained before conducting the study. This was a cross sectional study conducted in Travancore Medical College Hospital situated in Quilon district, Kerala. The study period was from January 2021 to June 2021. All patients from General medicine and Rheumatology department who were diagnosed with Rheumatoid arthritis, SLE and Primary Sjogren's syndrome in the Outpatient department were enrolled in the study. Patients aged between 30 and 65, who were diagnosed and confirmed cases of Rheumatoid arthritis, SLE and Primary Sjogren's syndrome were selected for the study. Patients who had a prior ocular surgery including cataract surgery were excluded from the study. Other exclusion criteria was ocular injury, use of contact lens history of smoking, radiation exposure, diagnosed episcleritis and scleritis.

Sampling method employed was Census method. Sample size accounted for 30 . All the study participants were asked detailed history and clinical examinations including the ophthalmology examinations was performed. Informed consent was obtained from the study participants. Visual acuity assessment using Snellen standard illuminated chart, Slit lamp examination including tests for dry eye and examination of fundus was performed on all the study participants. Data collected was entered in Microsoft Excel and descriptive statistics was reported as frequencies and percentages.

\section{RESULTS}

\begin{tabular}{|cccc|}
\hline & Age Category & Frequency & Percentage (\%) \\
\hline$\bullet$ & $<40$ years & 10 & 33.3 \\
$\bullet$ & $41-50$ years & 8 & 26.7 \\
$\bullet$ & $51-60$ years & 6 & 20 \\
$\bullet$ & $>$ >61 years & 6 & 20 \\
\hline & Table 1. Age Distribution of the Study Participants \\
\hline
\end{tabular}

The mean age of the study participants is $\mathbf{5 1}$ years (SD of 12.5) with minimum age being 36 years and maximum age being 78 years of age. Majority of the study participants were less than 40 years of age which accounted for $33.3 \%$ followed by age group of 41-50 years which accounted for $26.7 \%$. The study participants in the age group between 51 and 60 , and greater than 61 years accounted for $20 \%$ each. 


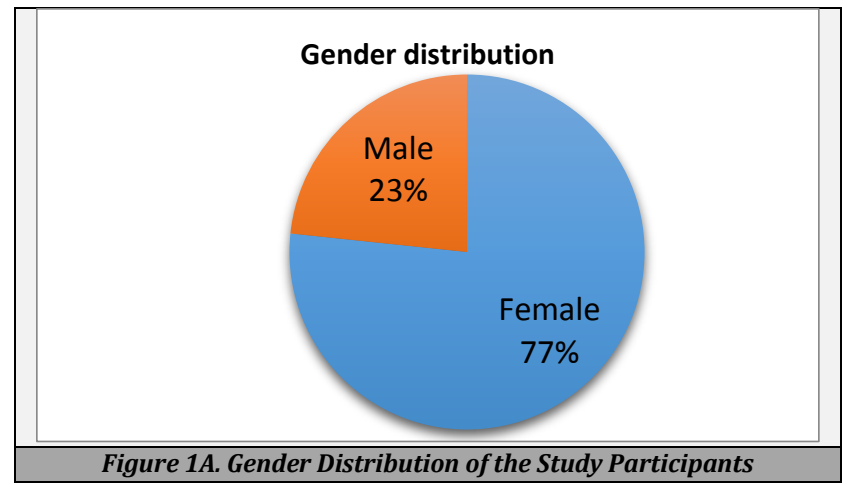

Majority of the study participants were female gender which was 23 in number which accounted for $77 \%$ and the rest 7 study participants were males, which accounted for $23 \%$

\begin{tabular}{|cccc|}
\hline & Diagnosis & Frequency & Percentage (\%) \\
• & Rheumatoid arthritis & 21 & 70 \\
- & Systemic Lupus Erythematosis & 5 & 16.7 \\
\hline Table 2. Distribution of the Auto Immune Disease among the Study & Participants $(\mathbf{N}=\mathbf{3 0})$ & \\
\hline
\end{tabular}

Twenty one study participants were diagnosed with Rheumatoid arthritis which accounted for $70 \%$. Five study participants were diagnosed with SLE, which accounted for $16.7 \%$ and the remaining four study participants had Primary SS which accounted for $13.3 \%$.

\begin{tabular}{|ccc|}
\hline $\begin{array}{c}\text { Grades of Conjunctival } \\
\text { Congestion }\end{array}$ & Frequency (n) & Percentage (\%) \\
$\bullet \quad$ No congestion & 15 & 50 \\
$\bullet \quad$ Mild congestion & 7 & 23 \\
- Moderate congestion (++) & 5 & 17 \\
• Severe congestion (+++) & 3 & 10 \\
\hline Table 3. Prevalence of Conjunctival Congestion among the Study \\
Participants (N=30)
\end{tabular}

Among the 30 study participants, fifteen had no conjunctival congestion which accounted for $50 \%$. Seven study participants had mild congestion which accounted for $23 \%$. Five study participants presented with moderate congestion which accounted for $17 \%$ and the remaining three study participants had severe conjunctival congestion which accounted for $10 \%$

\begin{tabular}{|cccc|}
\hline Grades of Conjunctival Staining & Frequency & Percentage \\
$\bullet$ & None & 13 & 43.3 \\
$\bullet$ & Variable & 6 & 20 \\
$\bullet$ & Moderate & 6 & 20 \\
$\bullet$ & Marked & 3 & 10 \\
Table 4. Conjunctival Staining in the Study Population $(\boldsymbol{N}=\mathbf{3 0})$ \\
\hline
\end{tabular}

There was no conjunctival staining in $43.3 \%$ of the study population. There was variable and moderate conjunctival staining in $20 \%$ each of the study participants. Marked conjunctival staining was present in $10 \%$ of the study participants. Moderate marked conjunctival staining was noted in the remaining $6.7 \%$ of the study population.

\begin{tabular}{|ccccccc|}
\hline Diagnosis & \multicolumn{2}{c|}{ Right Eye } & \multicolumn{2}{c|}{ Left Eye } & \multicolumn{2}{c|}{ Both Eyes } \\
- & s1 & s2 & s1 & s2 & s1 & s2 \\
Rheumatoid arthritis & 10 & 8 & 9 & 7 & 16 & 12 \\
• SLE & 16 & 13 & 17 & 14 & 23 & 17 \\
• Primary SS & 6 & 4.5 & 6 & 11 & 5 & 3 \\
\hline Table 5. Schirmer Tests (s1 and $\boldsymbol{s} 2)$ & of & the Right & Eye (RE), Left Eye \\
(LE)and Both Eyes (BE)of the Study Participants & \\
\hline
\end{tabular}

Schirmer test explains the severity of dry eye disease. The lesser the schirmer test value, more severe will be the dry eyed disease. Table 5 clearly shows the lower level of schirmer tests (s1 and s2) among the primary SS followed by the Rheumatoid arthritis group. Schrimer test was found normal in the study participants diagnosed with SLE.

\begin{tabular}{|c|c|c|c|c|c|}
\hline \multirow{2}{*}{ Diagnosis } & \multicolumn{5}{|c|}{ Tear Meniscus Height } \\
\hline & Frequency & Median & IQR & Minimum & Maximum \\
\hline Rheumatoid arthritis & 21 & 1.2 & 1.6 & 0 & 2 \\
\hline - SLE & 5 & 2 & 1 & 1 & 2 \\
\hline - $\quad$ PSS & 4 & .25 & .75 & 0 & 1 \\
\hline
\end{tabular}

Tear meniscus height is found to be significantly lower in the patients diagnosed with Primary Sjogrens syndrome (PSS) which indicates that the severity of dry eye disease is higher among the the PSS patients. Study participants diagnosed with SLE have higher tear meniscus height, which indicates less incidence of the dry eye disease. Overall, the tear meniscus height among the participants diagnosed with Primary SS and RA was found to be less than the participants diagnosed with SLE.

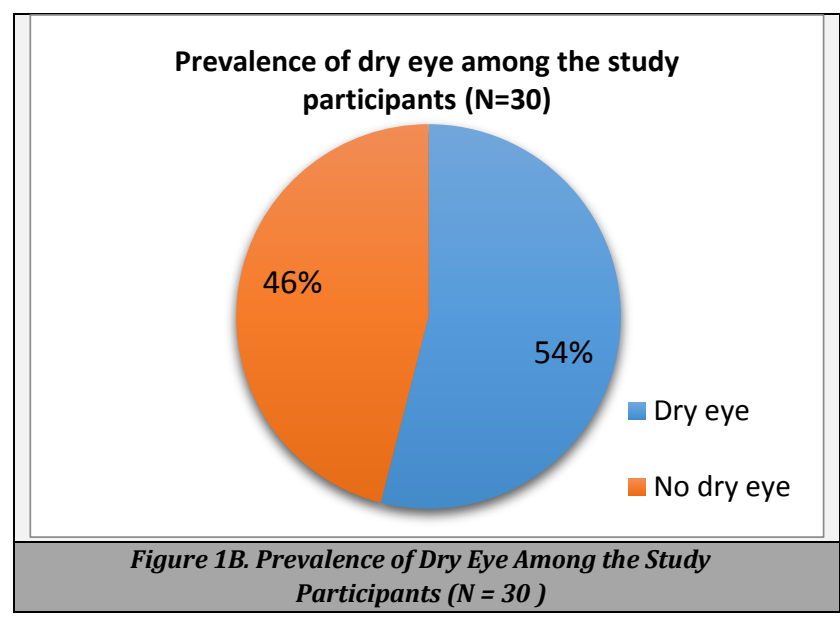

Fifty four percentages of the study participants was diagnosed to have dry eye disease among the study participants.

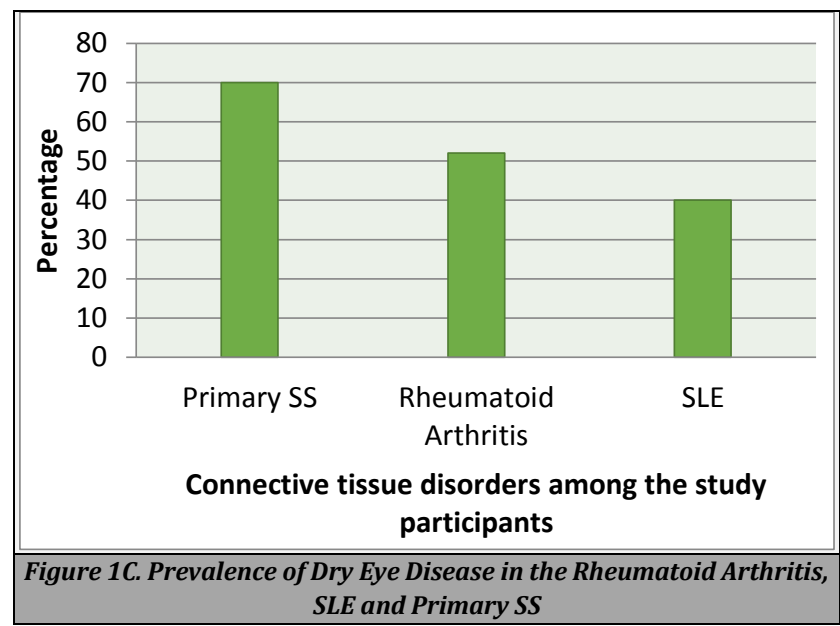

The prevalence of dry eye disease was found to be highest in the Primary SS, which accounted for $70 \%$ followed by Rheumatoid arthritis group of study participants which accounted for $52 \%$. The prevalence of dry eyes was found to 
be minimum in the study participants who has SLE, which accounted for $40 \%$.

\begin{tabular}{|c|c|c|c|c|}
\hline \multirow[b]{2}{*}{ Diagnosis } & \multicolumn{4}{|c|}{ Severity of Dry eye } \\
\hline & $\begin{array}{l}\text { Mild } \\
(\%)\end{array}$ & $\begin{array}{c}\text { Moderate } \\
\text { (\%) }\end{array}$ & $\begin{array}{c}\text { Severe } \\
\text { (\%) }\end{array}$ & $\begin{array}{c}\text { Very severe } \\
\text { (\%) }\end{array}$ \\
\hline - Rheumatoid arthritis & 50 & 28 & 20 & 2 \\
\hline$\bullet \quad$ SLE & 70 & 30 & 0 & 0 \\
\hline - Primary Sjogrens syndrome & 30 & 35 & 30 & 5 \\
\hline
\end{tabular}

The above table implies that the severity of dry eye disease is highest in Primary SS and the least in Rheumatoid arthritis. $35 \%$ of the study participants who had Primary SS had either severe or very severe dry eye disease; $22 \%$ of the study participants who were diagnosed to have Rheumatoid arthritis had severe to very severe dry eye disease. Whereas none of patient diagnosed to have SLE had dry eye disease.

\section{DISCUSSION}

Dry eye disease is a multifactorial condition that disrupts the tear film as well as ocular surface due to irregularities in the quantity and quality of the tear film which is commonly seen in systemic autoimmune diseases.

The prevalence of dry eye disease in our study was $54 \%$. In a study conducted by Yogeshwari et al conducted at Tamil nadu, it was observed that the prevalence of dry eye was 36 $\%$. The decreased prevalence can be attributed to increased sample size when compared to our study. Majority of the study participants $(90 \%)$ enrolled in the study were females in the study conducted by Yogeswari et al. This was similar to our study; where $77 \%$ of the study participants were females. This can be due to the menstrual irregularities, as it can cause changes in the ocular surface mainly due to hormonal factors.

In our study, the prevalence of dry eye disease was higher in the patients who have Primary Sjogrens syndrome which accounted for $70 \%$ followed by Rheumatoid arthritis and was least in the patients with SLE. In a study conducted by Kassan et al, the prevalence of Primary Sjogrens syndrome was found to be $68 \%$. The results were similar to our study findings. Among connective tissue disorders, Primary Sjogren's syndrome has the greater risk in causation of dry eye syndrome and the severity of the dry eye disease tend to be higher in the same group. Hence it is suggested that all the connective tissue disorders especially Primary Sjogrens syndrome must be screened for dry eye disease as soon as the diagnosis is confirmed from the medicine or rheumatology outpatient department. This can significantly reduce the visual morbidity.

From our study findings, patients diagnosed with SLE had less prevalence of dry eye disease compared to patients with other connective tissue diseases and the severity of the disease was also least in patients diagnosed with SLE. The prevalence of dry eye disease among SLE patients was found to be $40 \%$ in our study. This was similar to a study conducted by Silpa-Archa et al ${ }^{11}$ in which the prevalence of dry eye disease among the SLE patients were 34\%. Even though the severity especially the prevalence of severe and very severe cases of dry eye are less in SLE group, the milder and moderate dry eye disease cases among SLE patients need to be evaluated and treated as it has the chance of causing troublesome manifestations if left untreated.

Our study findings suggest that all the patients diagnosed and confirmed to be having connective tissue disorders from the general medicine or rheumatology departments must be immediately screened for dry eye disease in the ophthalmology department and should be evaluated based on the signs, symptoms and severity of the disease. If dry eye disease is diagnosed, the patients must be treated early at the initial stages to halt the progress of the disease to advanced stage and thereby reducing the visual morbidity as well.

\section{CONCLUSIONS}

From our study results and findings of related studies, it has been understood that the prevalence of Dry eye disease among the connective tissue disorders like Primary sjogren syndrome and Rheumatoid arthritis are relative high. Severity of the disease was also found higher in the connective tissue disorders. It is often under diagnosed due to the decreased symptoms at the initial stage. Patient usually present to the outpatient department at an advanced stage when the symptoms like eye irritation, redness, blurring of vision and burning of vision becomes troublesome. In majority of the patients who present at an advanced stage, ocular surface damage would have happened which lead to increased visual morbidity. Early screening of the patients who are suffering from connective tissue disorders at ophthalmology outpatient department in view of dry eye disease can aid in early diagnosis and improve the cure rate of the disease and decrease the ocular morbidity as well. The limitation of the present study was less sample size. Only 30 study participants were enrolled in this study which was due to less patients attending the outpatient department due to the high cases attributing to COVID 19 scenario in the state during the study period. A cross sectional study with large and adequate sample size or a systematic review of studies with similar objectives conducted across different health care settings is recommended for better in depth knowledge and understanding of the multifactorial condition.

Data sharing statement provided by the authors is available with the full text of this article at jemds.com.

Financial or other competing interests: None.

Disclosure forms provided by the authors are available with the full text of this article at jemds.com.

\section{REFERENCES}

[1] Letup MA, Baudouin C, Baum J. The definition and classification of dry eye disease: report of the Definition and Classification Subcommittee of the International Dry Eye Workshop. Ocul Surf 2007;5(2):75-92.

[2] McGinnigle S, Naroo SA, Eperjesi F. Evaluation of dry eye. Surv Ophthalmol 2012;57(4):293-316.

[3] Yogeswari A, Sivathanu SB, Rajalakshmi A, et al. Association of dry eyes with connective tissue disorder. International Journal of Scientific Study 2016;4(6):47-9. 
[4] Goren MB, Goren SB. Diagnostic tests in patients with symptoms of keratoconjunctivitis sicca. Am J Ophthalmol 1988;106(5):570-4.

[5] Kassan SS, Moutsopoulos HM. Clinical manifestations and early diagnosis of Sjögren syndrome. Arch Inter Med 2004;164(12):1275-84.

[6] Gonzales JA, Lietman TM. Ocular involvement in Sjögren's syndrome: advances in therapy. Current Treatment Options in Rheumatology 2018;4(1):99-109.

[7] Lemp MA. Dry eye (keratoconjunctivitis sicca), rheumatoid arthritis, and Sjögren's syndrome. Am J Ophthalmol 2005;140(5):898-9.

[8] Gipson IK, Argüeso P, Beuerman R, et al. Research in dry eye: report of the Research Subcommittee of the
International Dry Eye WorkShop (2007). Ocul Surf 2007;5(2):179-93.

[9] Gold DH, Morris DA, Henkind P. Ocular findings in systemic lupus erythematosus. $\mathrm{Br} \quad \mathrm{J}$ Ophthalmol 1972;56(11):800.

[10] Al-Bedri K, Al-Quriashi NK, Gorial FI, et al. Ocular manifestations in rheumatoid arthritis: a descriptive cross-sectional study from Iraq. Int J Sci Stud 2015;3(8):61-6.

[11] Silpa-Archa S, Lee JJ, Foster CS. Ocular manifestations in systemic lupus erythematosus. $\mathrm{Br} \mathrm{J}$ Ophthalmol 2016;100(1):135-41. 